PROCEEDINGS OF THE

AMERICAN MATHEMATICAL SOCIETY

Volume 131, Number 1, Pages 243-252

S 0002-9939(02)06552-8

Article electronically published on June 5, 2002

\title{
ON APPROXIMATELY CONVEX FUNCTIONS
}

\author{
ZSOLT PÁLES
}

(Communicated by Jonathan M. Borwein)

\begin{abstract}
A real valued function $f$ defined on a real interval $I$ is called $(\varepsilon, \delta)$-convex if it satisfies

$f(t x+(1-t) y) \leq t f(x)+(1-t) f(y)+\varepsilon t(1-t)|x-y|+\delta \quad$ for $x, y \in I, t \in[0,1]$.

The main results of the paper offer various characterizations for $(\varepsilon, \delta)$-convexity. One of the main results states that $f$ is $(\varepsilon, \delta)$-convex for some positive $\varepsilon$ and $\delta$ if and only if $f$ can be decomposed into the sum of a convex function, a function with bounded supremum norm, and a function with bounded Lipschitzmodulus. In the special case $\varepsilon=0$, the results reduce to that of Hyers, Ulam, and Green obtained in 1952 concerning the so-called $\delta$-convexity.
\end{abstract}

\section{INTRODUCTION}

The stability theory of functional inequalities started with the paper [HU52] of Hyers and Ulam who introduced the notion of $\delta$-convex function: If $D$ is a convex subset of a real linear space $X$ and $\delta$ is a nonnegative number, then a function $f: D \rightarrow \mathbb{R}$ is called $\delta$-convex if

$$
f(t x+(1-t) y) \leq t f(x)+(1-t) f(y)+\delta
$$

for all $x, y \in D, t \in[0,1]$. The basic result obtained by Hyers and Ulam states that if the underlying space $X$ is of finite dimension, then $f$ can be written as $f=g+h$, where $g$ is a convex function and $h$ is a bounded function whose supremum norm is not larger than $k_{n} \delta$, where the positive constant $k_{n}$ depends only on the dimension $n$ of the underlying space $X$. Hyers and Ulam proved that $k_{n} \leq(n(n+3)) /(4(n+1))$. Green Gre52, Cholewa Cho84] obtained much better estimations of $k_{n}$ showing that asymptotically $k_{n}$ is not bigger than $\left(\log _{2}(n)\right) / 2$. Laczkovich [Lac99] compared this constant to several other dimension-depending stability constants and proved that it is not less than $\left(\log _{2}(n / 2)\right) / 4$. This result shows that there is no stability results for infinite dimensional spaces $X$. A counterexample in this direction was earlier constructed by Casini and Papini [CP93]. The stability aspects of $\delta$-convexity are discussed by Ger Ger94.

If $t=1 / 2$ and (1) holds for all $x, y \in D$, then $f$ is called a $\delta$-Jensen-convex function. There is no analogous decomposition for $\delta$-Jensen-convex functions by

Received by the editors April 2, 2001 and, in revised form, September 4, 2001.

2000 Mathematics Subject Classification. Primary 26A51, 26B25.

Key words and phrases. Convexity, $(\varepsilon, \delta)$-convexity, stability of convexity, $(\varepsilon, \delta)$-subgradient, $(\varepsilon, \delta)$-subdifferentiability.

This research was supported by the Hungarian Scientific Research Fund (OTKA) Grant T038072 and by the Higher Education, Research and Development Fund (FKFP) Grant 0215/2001. 
the counterexample given by Cholewa [Cho84]. However, one can get BernsteinDoetsch type regularity theorems which show that $\delta$-Jensen-convexity and local upper boundedness imply $2 \delta$-convexity. This result is due to Bernstein and Doetsch [BD15] for $\delta=0$, and to $\mathrm{Ng}$ and Nikodem [NN93] in the case $\delta \geq 0$. For some recent extensions of these results to more general convexity concepts, see Pál00. For locally upper bounded $\delta$-Jensen-convex functions one can obtain the existence of an analogous stability constant $j_{n}$ (defined similarly as $k_{n}$ above). The sharp value of this stability constant has recently been found by Dilworth, Howard, and Roberts [DHR99] who have shown that

$$
j_{n}=\frac{1}{2}\left(\left[\log _{2}(n)\right]+1+\frac{n}{2^{\left[\log _{2}(n)\right]}}\right) \leq 1+\frac{1}{2} \log _{2}(n)
$$

is the best possible value for $j_{n}$. (Here [.] denotes the integer-part function.) The connection between $\delta$-Jensen-convexity and $\delta$ - $\mathbb{Q}$-convexity has been investigated by Mrowiec Mro01.

If $D \subset \mathbb{R}$ and (1) is supposed to be valid for all $x, y \in D$ except a set of 2dimensional Lebesgue measure zero, then one can speak about almost $\delta$-convexity. Results in this direction are due to Kuczma Kuc70 (the case $\delta=0$ ) and Ger Ger88 (the case $\delta \geq 0$ ).

Roughly speaking, the content of the Hyers-Ulam theorem can be expressed in the following way: A function $f$ is a perturbation of a convex function by a bounded function if and only if, for some $\delta$, it satisfies (11). Therefore, the presence of the error term $\delta$ refers to a bounded perturbation. In what follows, we consider more general perturbations of convex functions. We assume that $X$ is a normed space and we take, as a perturbation, functions of the form $h+\ell$, where $h$ is bounded and $\ell$ has a bounded Lipschitz-modulus. The investigation of such functions leads to the following stability notion: Let $\varepsilon, \delta \geq 0$ be constants and let $D$ be a subset of $X$. A function $f: D \rightarrow \mathbb{R}$ is called $(\varepsilon, \delta)$-convex on $D$ if

$$
f(t x+(1-t) y) \leq t f(x)+(1-t) f(y)+\varepsilon t(1-t)\|x-y\|+\delta
$$

for $x, y \in D, t \in[0,1]$. It is not difficult to see that if $X$ is a normed space, $f$ is of the form $f=g+h+\ell$, where $g$ is convex, $h$ is bounded with supremum norm not greater than $\delta / 2$, and the Lipschitz-modulus of $\ell$ defined by

$$
\operatorname{Lip}(\ell):=\inf \{L|| \ell(x)-\ell(y) \mid \leq L\|x-y\| \text { for } x, y \in D\}
$$

is not greater than $\varepsilon / 2$, then $f$ satisfies (2). The main result of this paper (see Corollary 4 below) is the more surprising statement that if $D$ is one dimensional (i.e., $D$ is a real interval) and $f$ satisfies (2), then $f$ admits a decomposition described above. Thus, one can observe, that the error terms $\varepsilon t(1-t)\|x-y\|$ and $\delta$ in (2) correspond to perturbations by Lipschitz and by bounded functions, respectively.

Most of the results obtained in this paper essentially use the fact that $D$ is one dimensional. Thus, the analogous problem for the higher dimensional setting remains unsolved.

\section{MAin Results}

Throughout the rest of this paper, we assume that $I$ is a nonempty open real interval of $\mathbb{R}$. Our first result offers a number of equivalent conditions for $(\varepsilon, \delta)$ convexity. 
Theorem 1. Let $f: I \rightarrow \mathbb{R}$ and $\varepsilon, \delta$ be nonnegative numbers. Then the following conditions are pairwise equivalent:

(i) $f$ is $(\varepsilon, \delta)$-convex on $I$, i.e.,

$$
f(t x+(1-t) y) \leq t f(x)+(1-t) f(y)+\varepsilon t(1-t)|x-y|+\delta
$$

for $x, y \in I, t \in[0,1]$.

(ii) For $x, u, y \in I$ with $x<u<y$,

$$
\frac{f(x)+\delta-f(u)}{x-u} \leq \frac{f(y)+\delta-f(u)}{y-u}+\varepsilon .
$$

(iii) There exists a function $p: I \rightarrow \mathbb{R}$ such that, for $x, u \in I$,

$$
f(u)+p(u)(x-u) \leq f(x)+\frac{\varepsilon}{2}|x-u|+\delta .
$$

(iv) If $x_{1}, \ldots, x_{n} \in I, t_{1}, \ldots, t_{n} \geq 0, t_{1}+\cdots+t_{n}=1$ and $u:=t_{1} x_{1}+\cdots+t_{n} x_{n}$, then

$$
f(u) \leq t_{1} f\left(x_{1}\right)+\cdots+t_{n} f\left(x_{n}\right)+\frac{\varepsilon}{2}\left(t_{1}\left|x_{1}-u\right|+\cdots+t_{n}\left|x_{n}-u\right|\right)+\delta .
$$

Proof. (i) $\Rightarrow($ ii): Assume that $f$ is $(\varepsilon, \delta)$-convex and let $x<u<y$ be arbitrary elements of $I$. Choose $t \in[0,1]$ such that $u=t x+(1-t) y$, that is let $t=$ $(y-u) /(y-x)$. Then applying the $(\varepsilon, \delta)$-convexity of $f$, we get

$$
f(u) \leq \frac{y-u}{y-x} f(x)+\frac{u-x}{y-x} f(y)+\varepsilon \frac{(y-u)(u-x)}{y-x}+\delta,
$$

which is equivalent to (4).

(ii) $\Rightarrow$ (iii): Assume that (ii) holds and define

$$
p(u):=\sup _{x \in I, x<u}\left(\frac{f(x)+\delta-f(u)}{x-u}-\frac{\varepsilon}{2}\right) \quad \text { for } \quad u \in I .
$$

Then, due to (ii), we have

$$
\frac{f(x)+\delta-f(u)}{x-u}-\frac{\varepsilon}{2} \leq p(u) \leq \frac{f(y)+\delta-f(u)}{y-u}+\frac{\varepsilon}{2}
$$

for all $x<u<y$ in $I$. The left-hand side inequality in (7) yields (5) in the case $x<u$, and analogously, the right-hand side inequality reduces to (15) in the case $x>u$. The case $x=u$ is obvious.

(iii) $\Rightarrow$ (iv): To deduce (iv) from (iii), let $x_{1}, \ldots, x_{n} \in I, t_{1}, \ldots, t_{n} \geq 0, t_{1}+\cdots+$ $t_{n}=1$ and $u:=t_{1} x_{1}+\cdots+t_{n} x_{n}$. Then, substituting $x$ by $x_{i}$ in (5), multiplying this inequality by $t_{i}$, and adding up the inequalities so obtained, we get

$$
\begin{aligned}
f(u) & =\sum_{i=1}^{n} t_{i}\left[f(u)+p(u)\left(x_{i}-u\right)\right] \\
& \leq \sum_{i=1}^{n} t_{i}\left(f\left(x_{i}\right)+\frac{\varepsilon}{2}\left|x_{i}-u\right|+\delta\right)=\sum_{i=1}^{n} t_{i} f\left(x_{i}\right)+\frac{\varepsilon}{2} \sum_{i=1}^{n} t_{i}\left|x_{i}-u\right|+\delta
\end{aligned}
$$

which is the desired inequality (6).

(iv) $\Rightarrow(\mathrm{i})$ : Taking $x_{1}=x, x_{2}=y, t_{1}=t$, and $t_{2}=1-t$ in condition (iv), one can immediately see that the inequality (6) reduces to (3). 
Remark 1. The equivalence of the conditions (i)-(iv) established in Theorem 1 reduces to well-known characterizations of convexity (cf. Hardy-Littlewood-Pólya [HLP34, Roberts-Varberg [RV73], and Kuczma [Kuc85]).

Motivated by condition (iii) of Theorem 1 , a function $f: I \rightarrow \mathbb{R}$ is called $(\varepsilon, \delta)$ subdifferentiable at a point $u \in I$, if there exists a real number $p=p(u)$ such that (5) holds for all $x \in I$. The $(\varepsilon, \delta)$-subgradient of $f$ at $u$ is defined as the set of all values $p(u)$ such that (5i) is valid for $x \in I$ and is denoted by $\partial_{\varepsilon, \delta} f(u)$. Clearly,

$$
\begin{aligned}
\partial_{\varepsilon, \delta} f(u) & =\left\{p \in \mathbb{R} \mid \frac{f(x)+\delta-f(u)}{x-u}-\frac{\varepsilon}{2} \leq p \leq \frac{f(y)+\delta-f(u)}{y-u}+\frac{\varepsilon}{2}, x<u<y\right\} \\
& =\left[\sup _{x \in I, x<u} \frac{f(x)+\delta-f(u)}{x-u}-\frac{\varepsilon}{2}, \inf _{y \in I, y>u} \frac{f(y)+\delta-f(u)}{y-u}+\frac{\varepsilon}{2}\right] .
\end{aligned}
$$

In the case if $f$ is $(\varepsilon, \delta)$-subdifferentiable at each point of $I$ and if a nondecreasing function $p: I \rightarrow \mathbb{R}$ can be chosen such that (5) holds, then we say that $f$ is nondecreasingly $(\varepsilon, \delta)$-subdifferentiable on $I$.

The equivalence of conditions (i) and (iii) in Theorem 1 now can be expressed via the following result.

Corollary 1. Let $f: I \rightarrow \mathbb{R}$ and $\varepsilon, \delta$ be nonnegative numbers. Then $f$ is $(\varepsilon, \delta)$ convex on $I$ if and only if it is $(\varepsilon, \delta)$-subdifferentiable on $I$.

In our next result we obtain the decomposition of $(\varepsilon, \delta)$-convex functions in terms of an $(\varepsilon, 0)$-convex function and a function whose supremum norm is not larger than $\delta / 2$.

Theorem 2. Let $f: I \rightarrow \mathbb{R}$ and $\varepsilon, \delta$ be nonnegative numbers. Then $f$ is $(\varepsilon, \delta)$ convex on $I$ if and only if there exists an $(\varepsilon, 0)$-convex function $\varphi: I \rightarrow \mathbb{R}$ such that $\|f-\varphi\|=\sup _{I}|f-\varphi| \leq \delta / 2$.

Proof. Assume that there exists an $(\varepsilon, 0)$-convex function $\varphi$ such that $\|f-\varphi\| \leq \delta / 2$. Denote by $h$ the difference $f-\varphi$. Then we have

$$
\varphi(t x+(1-t) y) \leq t \varphi(x)+(1-t) \varphi(y)+\varepsilon t(1-t)|x-y|
$$

and

$$
\begin{aligned}
h(t x+(1-t) y) \leq & t h(x)+(1-t) h(y) \\
& +|h(t x+(1-t) y)|+t|h(x)|+(1-t)|h(y)| \\
\leq & t h(x)+(1-t) h(y)+\delta
\end{aligned}
$$

for all $x, y \in I$ and $t \in[0,1]$. Summing up these two inequalities, we get that $f$ is $(\varepsilon, \delta)$-convex.

To prove the converse, assume that $f$ is $(\varepsilon, \delta)$-convex and apply Theorem 1 . Then there exists a function $p: I \rightarrow \mathbb{R}$ such that (5) holds for all $x, u \in I$. Define

$$
\varphi(x):=\sup _{u \in I}\left(f(u)+p(u)(x-u)-\frac{\varepsilon}{2}|x-u|-\frac{\delta}{2}\right) \quad \text { for } \quad x \in I .
$$

Then, by (5), we have that $\varphi(x) \leq f(x)+\delta / 2$ for all $x \in I$. On the other hand, clearly $f(x)-\delta / 2 \leq \varphi(x)$. Thus, we get that $\|f-\varphi\| \leq \delta / 2$.

To complete the proof, it remains to show that $\varphi$ is $(\varepsilon, 0)$-convex. For, let $x, y \in I$ and $t \in[0,1]$ be fixed. Let $c>0$ be an arbitrary (small) positive number. Then, 
by the definition of $\varphi(t x+(1-t) y)$, there exists $u \in I$ such that

$$
\varphi(t x+(1-t) y)-c<f(u)+p(u)(t x+(1-t) y-u)-\frac{\varepsilon}{2}|t x+(1-t) y-u|-\frac{\delta}{2} .
$$

Thus,

$$
\begin{aligned}
& \varphi(t x+(1-t) y)-c \\
& <t\left(f(u)+p(u)(x-u)-\frac{\varepsilon}{2}|x-u|-\frac{\delta}{2}\right) \\
& +(1-t)\left(f(u)+p(u)(y-u)-\frac{\varepsilon}{2}|y-u|-\frac{\delta}{2}\right) \\
& +\frac{\varepsilon}{2} t(|x-u|-|t x+(1-t) y-u|) \\
& +\frac{\varepsilon}{2}(1-t)(|y-u|-|t x+(1-t) y-u|) \\
& \leq t \varphi(x)+(1-t) \varphi(y)+\frac{\varepsilon}{2} t(1-t)|x-y|+\frac{\varepsilon}{2}(1-t) t|x-y| .
\end{aligned}
$$

Taking the limit $c \rightarrow 0$, it follows from the above inequality that $\varphi$ is $(\varepsilon, 0)$-convex.

Remark 2. The case $\varepsilon=0$ of the above theorem is the stability theorem of convex functions obtained by Hyers and Ulam HU52] (see also Green [Gre52]). Their results, however, concern also the higher but finite dimensional case.

Corollary 2. Let $f: I \rightarrow \mathbb{R}$ and $\varepsilon, \delta$ be nonnegative numbers. Then $f$ is $(\varepsilon, \delta)$-subdifferentiable at each point of $I$ if and only if there exists an $(\varepsilon, 0)$-subdifferentiable function $\varphi: I \rightarrow \mathbb{R}$ such that $\|f-\varphi\| \leq \delta / 2$. In addition, for all $u \in I$,

$$
\partial_{\varepsilon, 0} \varphi(u) \subset \partial_{\varepsilon, \delta} f(u) .
$$

Proof. By Corollary $1, f$ is $(\varepsilon, \delta)$-subdifferentiable on $I$ if and only if it is $(\varepsilon, \delta)$ convex on $I$. This property, by the previous theorem, is equivalent to the condition that there exists an $(\varepsilon, 0)$-convex function $\varphi$ such that $\|f-\varphi\| \leq \delta / 2$. Applying Corollary 1 again, we see that this latter property is equivalent to the condition that there exists an $(\varepsilon, 0)$-subdifferentiable function $\varphi$ such that $\|f-\varphi\| \leq \delta / 2$.

To see the inclusion between the subdifferentials, let $u \in I$ and $p \in \partial_{\varepsilon, 0} \varphi(u)$. Then

$$
\varphi(u)+p(x-u) \leq \varphi(x)+\frac{\varepsilon}{2}|x-u| \quad \text { for } \quad x \in I .
$$

Using $\|f-\varphi\| \leq \delta / 2$, we get, for all $x \in I$, that

$f(u)+p(x-u) \leq \varphi(u)+p(x-u)+\frac{\delta}{2} \leq \varphi(x)+\frac{\varepsilon}{2}|x-u|+\frac{\delta}{2} \leq f(x)+\frac{\varepsilon}{2}|x-u|+\delta$, i.e., $p \in \partial_{\varepsilon, \delta} f(u)$.

Now we intend to give characterizations of $(\varepsilon, 0)$-subdifferentiable functions. First we investigate the properties of the $(\varepsilon, 0)$-subdifferential. For, introduce the following notion: A function $p: I \rightarrow \mathbb{R}$ is called $\varepsilon$-nondecreasing on $I$ if

$$
p(x) \leq p(y)+\varepsilon
$$

holds for all $x \leq y$ in $I$. A set-valued function $P: I \rightarrow 2^{\mathbb{R}}$ is called $\varepsilon$-nondecreasing if any selection function $p$ of $P$ is $\varepsilon$-nondecreasing, which is equivalent to requiring that $u \leq v+\varepsilon$ whenever $u \in P(x), v \in P(y)$, and $x \leq y$. 
The connection between $\varepsilon$-nondecreasing and nondecreasing functions is described in the next result.

Theorem 3. Let $I$ be an open interval of $\mathbb{R}, p: I \rightarrow \mathbb{R}$, and $\varepsilon$ be a nonnegative number. Then $p$ is $\varepsilon$-nondecreasing if and only if there exists a nondecreasing function $q: I \rightarrow \mathbb{R}$ such that $\|p-q\| \leq \varepsilon / 2$.

Proof. Assume that $q$ is nondecreasing such that $\|p-q\| \leq \varepsilon / 2$. Then for $x \leq y$, we have

$$
p(x) \leq q(x)+|p(x)-q(x)| \leq q(y)+\frac{\varepsilon}{2} \leq p(y)+\frac{\varepsilon}{2}+|p(y)-q(y)| \leq p(y)+\varepsilon .
$$

Thus, $p$ is $\varepsilon$-nondecreasing.

Conversely, assume that $p$ is $\varepsilon$-nondecreasing and define

$$
q(x):=\sup _{v \in I, v \leq x}\left(p(v)-\frac{\varepsilon}{2}\right) \quad \text { for } \quad x \in I .
$$

Then $q$ is obviously nondecreasing. By its definition, we have that

$$
p(x)-\frac{\varepsilon}{2} \leq q(x) .
$$

On the other hand, using that $p$ is $\varepsilon$-nondecreasing, $p(v) \leq p(x)+\varepsilon$ for all $v \leq x$, whence

$$
q(x)=\sup _{v \in I, v \leq x}\left(p(v)-\frac{\varepsilon}{2}\right) \leq p(x)+\frac{\varepsilon}{2} .
$$

The two inequalities obtained yield that $\|p-q\| \leq \varepsilon / 2$.

Corollary 3. Let $\varphi: I \rightarrow \mathbb{R}$ be an $(\varepsilon, 0)$-convex function on $I$, where $\varepsilon$ is a nonnegative number. Then $\varphi$ is nondecreasingly $(2 \varepsilon, 0)$-subdifferentiable on $I$.

Proof. By Corollary $1, \varphi$ is $(\varepsilon, 0)$-subdifferentiable on $I$, that is there exists a function $p: I \rightarrow \mathbb{R}$ such that

$$
\varphi(u)+p(u)(x-u) \leq \varphi(x)+\frac{\varepsilon}{2}|x-u| \quad \text { for } \quad x, u \in I .
$$

Interchanging $x$ and $u$ and adding up the two inequalities, we get

$$
(p(u)-p(x))(x-u) \leq \varepsilon|x-u| \quad \text { for } \quad x, u \in I .
$$

If $x<u$, then this inequality yields

$$
p(x)-p(u) \leq \varepsilon,
$$

whence it follows that $p$ is $\varepsilon$-nondecreasing. By the previous result, there exists a nondecreasing function $q: I \rightarrow \mathbb{R}$ such that $\|p-q\| \leq \varepsilon / 2$. Thus, using (8), we get

$$
\begin{aligned}
\varphi(u)+q(u)(x-u) & \leq \varphi(u)+p(u)(x-u)+|(p(u)-q(u))(x-u)| \\
& \leq \varphi(u)+p(u)(x-u)+\frac{\varepsilon}{2}|x-u| \\
& \leq \varphi(x)+\varepsilon|x-u|
\end{aligned}
$$

for all $x, u \in I$. Hence $\varphi$ is nondecreasingly $(2 \varepsilon, 0)$-subdifferentiable.

Now we characterize nondecreasingly $(\varepsilon, 0)$-subdifferentiable functions.

Theorem 4. Let $\varphi: I \rightarrow \mathbb{R}$ and $\varepsilon$ be a nonnegative number. Then $\varphi$ is nondecreasingly $(\varepsilon, 0)$-subdifferentiable on $I$ if and only if there exists a convex function $g: I \rightarrow \mathbb{R}$ such that $\operatorname{Lip}(\varphi-g) \leq \varepsilon / 2$. 
Proof. Assume that $\varphi=g+\ell$, where $g$ is convex and $\operatorname{Lip}(\ell) \leq \varepsilon / 2$. Then, by Corollary 3 (with $\varepsilon=0$ ) applied to $g$, there exists a nondecreasing function $q: I \rightarrow$ $\mathbb{R}$ such that

$$
g(u)+q(u)(x-u) \leq g(x) \quad \text { for } \quad x, u \in I .
$$

The function $\ell$ also satisfies

$$
\ell(u) \leq \ell(x)+\frac{\varepsilon}{2}|x-u| \quad \text { for } \quad x, u \in I .
$$

Adding up these inequalities, we get that $\varphi$ satisfies

$$
\varphi(u)+q(u)(x-u) \leq \varphi(x)+\frac{\varepsilon}{2}|x-u| \quad \text { for } \quad x, u \in I,
$$

i.e., it is nondecreasingly $(\varepsilon, 0)$-subdifferentiable.

Conversely, assume that $\varphi$ is nondecreasingly $(\varepsilon, 0)$-subdifferentiable. Then, we have that

$$
q(u)(x-u) \leq \varphi(x)-\varphi(u)+\frac{\varepsilon}{2}|x-u| \quad \text { for } \quad x, u \in I .
$$

Define now $g: I \rightarrow \mathbb{R}$ as the primitive function of $q$, that is, let $g(x):=\int_{x_{0}}^{x} q$, where $x_{0}$ is an arbitrarily fixed element of $I$. Then, $q$ being nondecreasing, we get that $g$ is a convex function. To complete the proof of the theorem, we show that the Lipschitz modulus of the function $\ell:=\varphi-g$ is not greater than $\varepsilon / 2$.

For, let $x<y, x, y \in I$ be arbitrary. Let $t_{0}=x<t_{1}<\cdots<t_{n}=y$ be an arbitrary division of the interval $[x, y]$. Substituting $x:=t_{i-1}, u:=t_{i}$ for $i=1, \ldots, n$ into (9) and adding the inequalities obtained, we get

$$
\begin{aligned}
\sum_{i=1}^{n} q\left(t_{i}\right)\left(t_{i-1}-t_{i}\right) & \leq \sum_{i=1}^{n}\left(\varphi\left(t_{i-1}\right)-\varphi\left(t_{i}\right)+\frac{\varepsilon}{2}\left(t_{i}-t_{i-1}\right)\right) \\
& =\varphi\left(t_{0}\right)-\varphi\left(t_{n}\right)+\frac{\varepsilon}{2}\left(t_{n}-t_{0}\right) \\
& =\varphi(x)-\varphi(y)+\frac{\varepsilon}{2}(y-x) .
\end{aligned}
$$

The left-hand side of this inequality is arbitrarily close to $-\int_{x}^{y} q=g(x)-g(y)$ if $\max _{1 \leq i \leq n}\left(t_{i}-t_{i-1}\right)$ is small enough. Therefore, we obtain

$$
g(x)-g(y) \leq \varphi(x)-\varphi(y)+\frac{\varepsilon}{2}(y-x),
$$

that is,

$$
\ell(y)-\ell(x) \leq \frac{\varepsilon}{2}(y-x) .
$$

On the other hand, substituting $x:=t_{i}, u:=t_{i-1}$ for $i=1, \ldots, n$ into (9) and adding these inequalities, we get

$$
\begin{aligned}
\sum_{i=1}^{n} q\left(t_{i-1}\right)\left(t_{i}-t_{i-1}\right) & \leq \sum_{i=1}^{n}\left(\varphi\left(t_{i}\right)-\varphi\left(t_{i-1}\right)+\frac{\varepsilon}{2}\left(t_{i}-t_{i-1}\right)\right) \\
& =\varphi\left(t_{n}\right)-\varphi\left(t_{0}\right)+\frac{\varepsilon}{2}\left(t_{n}-t_{0}\right) \\
& =\varphi(y)-\varphi(x)+\frac{\varepsilon}{2}(y-x),
\end{aligned}
$$

whence, by taking the limit, we get that

$$
g(y)-g(x)=\int_{x}^{y} q \leq \varphi(y)-\varphi(x)+\frac{\varepsilon}{2}(y-x) .
$$


This inequality yields

$$
\ell(x)-\ell(y) \leq \frac{\varepsilon}{2}(y-x),
$$

which, together with (10), results that

$$
|\ell(x)-\ell(y)| \leq \frac{\varepsilon}{2}(y-x)
$$

for all $x<y$ in $I$. Hence, the Lipschitz modulus of $\ell$ is not greater than $\varepsilon / 2$.

In view of Theorem 2 , in order to obtain the desired decomposition of $(\varepsilon, \delta)$ convex functions, it suffices to show that $(\varepsilon, 0)$-convex functions can be decomposed in terms of a convex function and a function with small Lipschitz modulus.

Theorem 5. Let $\varphi: I \rightarrow \mathbb{R}$ and $\varepsilon$ be a nonnegative number. If there exists a convex function $g: I \rightarrow \mathbb{R}$ such that $\operatorname{Lip}(\varphi-g) \leq \varepsilon / 2$, then $\varphi$ is $(\varepsilon, 0)$-convex on $I$.

Conversely, if $\varphi$ is $(\varepsilon, 0)$-convex on $I$, then there exists a convex function $g: I \rightarrow$ $\mathbb{R}$ such that $\operatorname{Lip}(\varphi-g) \leq \varepsilon$.

Proof. Assume that there exists a convex function $g: I \rightarrow \mathbb{R}$ such that, for $\ell:=$ $\varphi-g$, we have $\operatorname{Lip}(\ell) \leq \varepsilon / 2$. Then we have

$$
g(t x+(1-t) y) \leq t g(x)+(1-t) g(y)
$$

and

$$
\begin{aligned}
\ell(t x+ & (1-t) y) \\
\leq & t \ell(x)+(1-t) \ell(y) \\
& \quad+t|\ell(x)-\ell(t x+(1-t) y)|+(1-t)|\ell(y)-\ell(t x+(1-t) y)| \\
\leq & t \ell(x)+(1-t) \ell(y)+t \frac{\varepsilon}{2}(1-t)|x-y|+(1-t) \frac{\varepsilon}{2} t|x-y| \\
= & t \ell(x)+(1-t) \ell(y)+\varepsilon t(1-t)|x-y|
\end{aligned}
$$

for all $x, y \in I$ and $t \in[0,1]$. Summing up these two inequalities, we get that $\varphi$ is $(\varepsilon, 0)$-convex.

Conversely, assume that $\varphi$ is $(\varepsilon, 0)$-convex on $I$. Then, by Corollary 3 , it is also nondecreasingly $(2 \varepsilon, 0)$-subdifferentiable. Applying Theorem 4 (with $\varepsilon$ replaced by $2 \varepsilon$ ), we get that there exists a convex function $g: I \rightarrow \mathbb{R}$ satisfying the condition $\operatorname{Lip}(\varphi-g) \leq \varepsilon$.

Remark 3. There is a discrepancy in the above result, since it is not an if-and-onlyif characterization of $(\varepsilon, 0)$-convex functions. We show, however, that the constants in the result cannot be improved. Without loss of generality, we may assume that $[-1,1] \subset I$.

First let $\varphi(x):=-\varepsilon|x| / 2$ for $x \in I$. Then, obviously, $\operatorname{Lip}(\varphi-0)=\operatorname{Lip}(\varphi)=\varepsilon / 2$. Therefore, by the first part of Theorem $5, \varphi$ is $(\varepsilon, 0)$-convex, i.e., it satisfies

$$
\varphi(t x+(1-t) y) \leq t \varphi(x)+(1-t) \varphi(y)+\varepsilon t(1-t)|x-y| \quad(x, y \in I, t \in[0,1]) .
$$

Putting $x:=t-1, y:=t$ into this inequality, we can see that it holds with equality. Thus, $\varphi$ cannot be $(\bar{\varepsilon}, 0)$-convex for any $0 \leq \bar{\varepsilon}<\varepsilon$.

On the other hand, we show that there exists an $(\varepsilon, 0)$-convex function $\varphi: I \rightarrow \mathbb{R}$ such that $\operatorname{Lip}(\varphi-g) \geq \varepsilon$ for all convex functions $g: I \rightarrow \mathbb{R}$. Define $\varphi: I \rightarrow \mathbb{R}$ by

$$
\varphi(x):= \begin{cases}\varepsilon\left(1-x^{2}\right) / 2 & \text { if }|x|<1, \\ 0 & \text { if }|x| \geq 1 .\end{cases}
$$


In order to prove the $(\varepsilon, 0)$-convexity of $\varphi$, due to Theorem 1 , it suffices to check the inequality

$$
\frac{\varphi(x)-\varphi(u)}{x-u} \leq \frac{\varphi(y)-\varphi(u)}{y-u}+\varepsilon \quad(x, u, y \in I, x<u<y) .
$$

We distinguish three cases according to the position of $u$ with respect to the interval $[-1,1]$.

$$
\begin{aligned}
& \text { If } u \leq-1 \text {, then } \frac{\varphi(x)-\varphi(u)}{x-u}=0 \text { and } \\
& \frac{\varphi(y)-\varphi(u)}{y-u} \begin{cases}\leq \frac{\varphi(y)-\varphi(-1)}{y+1}=\frac{\varepsilon\left(1-y^{2}\right)}{2(y+1)}=\frac{\varepsilon(1-y)}{2} \leq \varepsilon & \text { if }|y|<1, \\
=0 & \text { if }|y| \geq 1 .\end{cases}
\end{aligned}
$$

Thus, (11) trivially holds.

A similar argument shows (11) also in the case when $u \geq 1$.

The last case is when $|u|<1$. Then, it is elementary to check the following inequalities and equalities:

$$
\frac{\varphi(x)-\varphi(u)}{x-u} \leq \frac{\varphi(u)-\varphi(-1)}{u-(-1)}=\frac{\varepsilon(1-u)}{2}=\frac{\varphi(u)-\varphi(1)}{u-1}+\varepsilon \leq \frac{\varphi(y)-\varphi(u)}{y-u}+\varepsilon .
$$

Therefore, (11) is also valid in this case.

Thus we have proved (11), i.e., the $(\varepsilon, 0)$-convexity of $\varphi$.

Finally, we show that $\operatorname{Lip}(\varphi-g) \geq \varepsilon$ for all convex functions $g: I \rightarrow \mathbb{R}$. Assume on the contrary, that $\lambda=\operatorname{Lip}(\varphi-g)<\varepsilon$ for some convex function $g$. Then the right (and also left) derivatives of $\ell=\varphi-g$ are between $-\lambda$ and $\lambda$, that is,

$$
-\lambda \leq \varphi^{\prime}(t)-g^{+}(t) \leq \lambda \quad(|t|<1) .
$$

Let $-1<x<y<1$ be arbitrary. Then, by the convexity of $g$, we have $g^{+}(x) \leq$ $g^{+}(y)$. Substituting $t=x$, and $t=y$ into the above inequality, we get

$$
-\lambda \leq \varphi^{\prime}(y)-g^{+}(y) \leq \varphi^{\prime}(y)-g^{+}(x) \leq \varphi^{\prime}(y)-\varphi^{\prime}(x)+\lambda=\varepsilon(x-y)+\lambda .
$$

Taking the limits $x \rightarrow-1$ and $y \rightarrow 1$, this inequality reduces to

$$
-\lambda \leq-2 \varepsilon+\lambda
$$

which yields $\varepsilon \leq \lambda$, a contradiction.

As an immediate consequence of Theorems 2 and 5, we get the main result of this paper.

Corollary 4. Let $f: I \rightarrow \mathbb{R}$ and $\varepsilon, \delta$ be nonnegative numbers. If $f$ is of the form $f=g+\ell+h$, where $g: I \rightarrow \mathbb{R}$ is convex, $h: I \rightarrow \mathbb{R}$ is bounded with $\|h\| \leq \delta / 2$, and $\ell: I \rightarrow \mathbb{R}$ is Lipschitz with Lip $(\ell) \leq \varepsilon / 2$, then $f$ is $(\varepsilon, \delta)$-convex on $I$.

Conversely, if $f$ is $(\varepsilon, \delta)$-convex on $I$, then there exist a convex function $g: I \rightarrow$ $\mathbb{R}$, a bounded function $h: I \rightarrow \mathbb{R}$ with $\|h\| \leq \delta / 2$, and a Lipschitz function $\ell: I \rightarrow \mathbb{R}$ with $\operatorname{Lip}(\ell) \leq \varepsilon$ such that $f=g+h+\ell$.

Proof. Assume that $f$ is of the form $f=g+h+\ell$. Then, by Theorem $5, \varphi=g+\ell$ is an $(\varepsilon, 0)$-convex function. Since $\|f-\varphi\| \leq \delta / 2$, hence, by Theorem $2, f$ is $(\varepsilon, \delta)$-convex.

Conversely, suppose that $f$ is $(\varepsilon, \delta)$-convex. Then, by Theorem 2 , there exists an $(\varepsilon, 0)$-convex function $\varphi: I \rightarrow \mathbb{R}$ such that, with $h:=f-\varphi$, we have $\|h\| \leq \delta / 2$. 
Now, applying Theorem 5 , there exists a convex function $g: I \rightarrow \mathbb{R}$ such that, with the notation $\ell:=\varphi-g$, we have $\operatorname{Lip}(\ell) \leq \varepsilon$. Thus we obtain

$$
f=\varphi+h=g+\ell+h,
$$

which is the desired decomposition.

\section{REFERENCES}

[BD15] F. Bernstein and G. Doetsch, Zur Theorie der konvexen Funktionen, Math. Annalen 76 (1915), 514-526.

[Cho84] P. W. Cholewa, Remarks on the stability of functional equations, Aequationes Math. 27 (1984), 76-86. MR 86d:39016

[CP93] E. Casini and P. L. Papini, A counterexample to the infinity version of the Hyers-Ulam stability theorem, Proc. Amer. Math. Soc. 118 (1993), 885-890. MR 93i:26022

[DHR99] S. J. Dilworth, R. Howard, and J. W. Roberts, Extremal approximately convex functions and estimating the size of convex hulls, Advances Math. 148 (1999), 1-43. MR 2001c:26015

[Ger88] R. Ger, Almost approximately convex functions, Math. Slovaca 38 (1988), no. 1, 61-78. MR 89m:26020a

[Ger94] R. Ger, Stability aspects of delta-convexity, Stability of mappings of Hyers-Ulam type (Palm Harbor, FL), Hadronic Press, Palm Harbor, FL, 1994, pp. 99-109. MR 95i:39026

[Gre52] J. W. Green, Approximately convex functions, Duke Math. J. 19 (1952), 499-504. MR 14:254c

[HLP34] G. H. Hardy, J. E. Littlewood, and G. Pólya, Inequalities, Cambridge University Press, Cambridge, 1934, (first edition), 1952 (second edition). MR 13:727e

[HU52] D. H. Hyers and S. M. Ulam, Approximately convex functions, Proc. Amer. Math. Soc. 3 (1952), 821-828. MR 14:254b

[Kuc70] M. Kuczma, Almost convex functions, Colloq. Math. 21 (1970), 279-284. MR 41:7043

[Kuc85] M. Kuczma, An Introduction to the Theory of Functional Equations and Inequalities, Państwowe Wydawnictwo Naukowe - Uniwersytet Śląski, Warszawa-KrakówKatowice, 1985. MR 86i:39008

[Lac99] M. Laczkovich, The local stability of convexity, affinity and of the Jensen equation, Aequationes Math. 58 (1999), 135-142. MR 2001d:39028

[Mro01] J. Mrowiec, Remark on approximately Jensen-convex functions, C. R. Math. Rep. Acad. Sci. Canada 23 (2001), 16-21.

[NN93] C. T. Ng and K. Nikodem, On approximately convex functions, Proc. Amer. Math. Soc. 118 (1993), no. 1, 103-108. MR 93f:26006

[Pál00] Zs. Páles, Bernstein-Doetsch-type results for general functional inequalities, Rocznik Nauk.-Dydakt. Prace Mat. 17 (2000), 197-206, Dedicated to Professor Zenon Moszner on his 70th birthday. MR 2001k:26015

[RV73] A. W. Roberts and D. E. Varberg, Convex Functions, Academic Press, New YorkLondon, 1973. MR 56:1201

Institute of Mathematics and Informatics, University of Debrecen, H-4010 Debrecen, PF. 12, Hungary

E-mail address: pales@math.klte.hu 